by décolletage, and it will not leave the cosmetic problem for a lady who cares about her physical appearance. More importantly, the procedures are executed off-pump. The second benefit is safety: capture of the dislocated occluder through the inferior partial median sternotomy is relatively easier than other approaches for perfect exposure. When the capture or the closure procedure is failed for some reason, the incision can be extended upward, and conventional surgical closure can be performed immediately.

To our knowledge, this is the first case in which a dislocated occluder has been captured without CPB and in which the peratrial device closure was performed after the failure of percutaneous occluder implantation. The case reminds us that capture of a dislocated occluder without $\mathrm{CPB}$ is technically feasible and that peratrial device closure might be a new direction for therapy of septal defects.

\section{References}

1. Fischer G, Stieh J, Uebing A, Hoffmann U, Morf G, Kramer HH. Experience with transcatheter closure of secundum atrial septal defects using the Amplatzer septal occluder: a single centre study in 236 consecutive patients. Heart. 2003;89: 199-204.

2. Fu Y-C, Cao Q-L, Hijazi ZM. Device closure of large atrial septal defects: technical considerations. J Cardiovasc Med (Hagerstown). 2007;8:30-3.

3. Hongxin L, Wenbin G, Lijun S, Zhengjun W, Hao L, Chengwei Z, et al. Intraoperative device closure of secundum atrial septal defect with a right anterior minithoracotomy in 100 patients. J Thorac Cardiovasc Surg. 2007;134:946-51.

4. Berdat PA, Chatterjee T, Pfammatter JP, Windecker S, Meier B, Carrel T. Surgical management of complications after transcatheter closure of an atrial septal defect or patent foramen ovale. J Thorac Cardiovasc Surg. 2000;120:1034-9.

5. Thomson JDR, Aburawi EH, Watterson KG, Van Doorn C, Gibbs JL. Surgica and transcatheter (Amplatzer) closure of atrial septal defects: a prospective comparison of results and cost. Heart. 2002;87:466-9.

\title{
Stent graft coarctation after frozen elephant trunk procedure: An unusual complication
}

\author{
Davide Pacini, MD, ${ }^{\mathrm{a}}$ Alessandro Armaro, MD, ${ }^{\mathrm{a}}$ Luca Di Marco, MD, ${ }^{\mathrm{a}}$ Vincenzo Russo, MD, ${ }^{\mathrm{b}}$ and Roberto Di Bartolomeo, MD, \\ Bologna, Italy
}

\section{Supplemental material is available online.}

Recently, a hybrid repair combining endovascular treatment with conventional surgery, known as the "frozen elephant trunk" technique, ${ }^{1}$ has been described as a single-stage procedure for the treatment of diffuse pathology of the thoracic aorta. We report an interesting case of a patient with Marfan syndrome who underwent frozen elephant trunk repair in which coarctation of the endoprosthesis was postoperatively diagnosed and successfully treated with another endovascular procedure.

\section{CLINICAL SUMMARY}

A 52-year-old male patient with Marfan syndrome and severe scoliosis leading to severe respiratory insufficiency

\footnotetext{
From the Departments of Cardiac Surgery and Radiology, ${ }^{\mathrm{b}}$ S Orsola-Malpighi Hospital, University of Bologna, Bologna, Italy.

Received for publication Jan 24, 2008; accepted for publication March 22, 2008.

Address for reprints: Davide Pacini, MD, Unità Operativa di Cardiochirurgia, Università degli studi di Bologna, Policlinico S Orsola, Via Massarenti, 9, 40138 Bologna, Italia (E-mail: dpacini@hotmail.com).

J Thorac Cardiovasc Surg 2009;137:1027-9

$0022-5223 / \$ 36.00$

Copyright $(c) 2009$ by The American Association for Thoracic Surgery

doi:10.1016/j.jtcvs.2008.03.036
}

(Figure 1, A) and previous mitral valve replacement had an acute type B aortic dissection that was medically treated. Six months later, the patient was referred to our department because of rapid dilatation of the false lumen of the descending thoracic aorta, which presented a severe tortuosity, facing the spinal column scoliosis (Figure 1, $B$ and Figure E1, Al-A4). The ascending aorta and the aortic root were dilated, and moderate aortic valve regurgitation was demonstrated. The patient underwent aortic root replacement with a composite valve graft together with aortic arch and descending aorta replacement in a frozen elephant trunk procedure. Cardiopulmonary bypass was established through the right axillary artery and the right atrium. The cerebral protection method consisted of antegrade selective cerebral perfusion and mild hypothermia. ${ }^{2}$ Myocardial protection was achieved with cold crystalloid cardioplegia. The stent graft system (E-vita open; Jotec Inc, Hechingen, Germany) was introduced in an antegrade manner in the true lumen of the descending aorta over a stiff guide wire previously positioned under transesophageal echocardiographic control, through the right femoral artery. The proximal landing zone was approximately $4 \mathrm{~cm}$ distal to the left subclavian artery.

Weaning from cardiopulmonary bypass was uneventful, as was the initial postoperative course. A computed tomographic scan on postoperative day 9 revealed severe kinking 

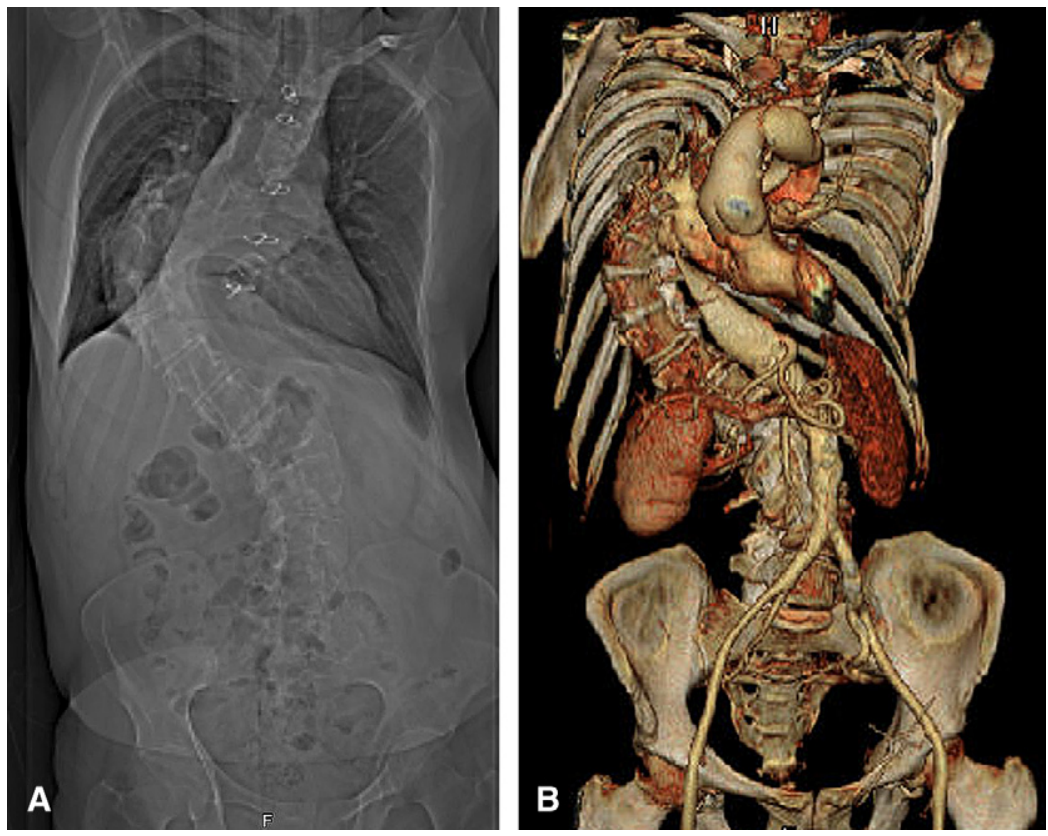

FIGURE 1. Multidetector computed tomographic scout (A) and volume rendering (B) images showing severe right convex vertebral scoliosis together with significant kinking of descending thoracic aorta.

of the stent graft leading to coarctation (Figure 2, $A$, and Figure E1, B1-B4). At that time, a pressure gradient of $52 \mathrm{~mm} \mathrm{Hg}$ was measured between the radial and femoral arteries. The patient underwent an endovascular procedure, and a Medtronic Valiant thoracic stent graft (Medtronic,
Inc, Minneapolis, Minn) was successfully placed inside the E-vita open graft. After ballooning of the new endoprosthesis, a good angiographic result was achieved, with the disappearance of the arterial pressure gradient. The postoperative course was uneventful. The predischarge computed
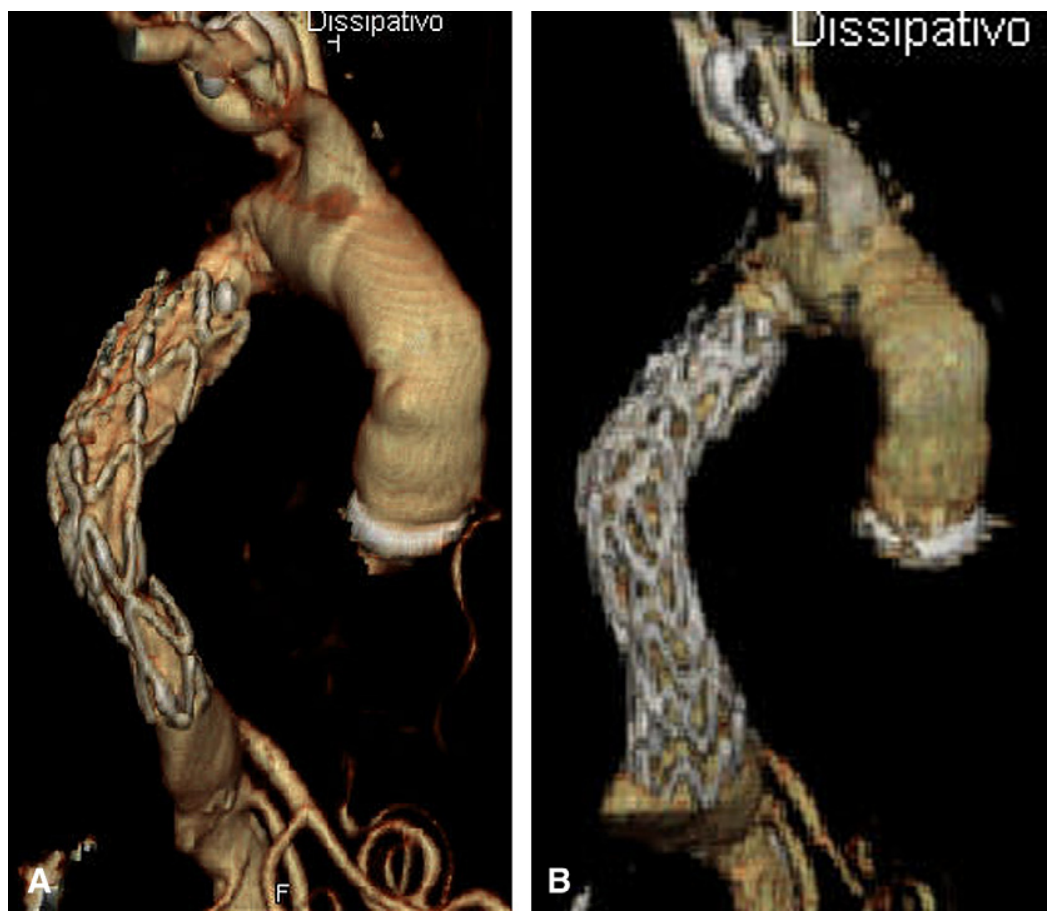

FIGURE 2. Multidetector computed tomographic volume rendering images showing descending thoracic aorta after surgical correction with E-vita open prosthesis (A) and after endovascular stent graft extension (B). 
tomographic scan revealed improvement of the endoprosthetic kinking and a partially thrombosed false lumen (Figure 2, B, and Figure E1, Cl-C4). At the 6-month follow-up, a computed tomographic scan showed excellent postoperative results, demonstrating total exclusion of the aneurysms and a thrombosed perigraft space around the frozen part of the trunk.

\section{DISCUSSION}

We describe here an interesting case of a patient with Marfan syndrome affected by a postdissection aneurysm of the descending thoracic aorta and dilatation of the ascending aorta. The anatomy of the descending thoracic aorta was unusual; the aorta had a pronounced tortuosity that followed the severe spinal column scoliosis. Traditionally, these kinds of complex thoracic aneurysms are treated with the elephant trunk technique ${ }^{1}$; however, this staged approach is associated with considerable overall mortality and morbidity. ${ }^{3}$ In our case, this approach was not considered possible because of a severe preoperative pulmonary insufficiency, which contraindicated thoracotomy. On the other hand, an endovascular procedure, a possible alternative method for the second-stage aortic repair, was not considered because passage through the extremely contorted aorta could have been very dangerous and difficult. The frozen elephant trunk technique was therefore considered to be the safest and the best repair technique for this specific patient. The operation was successfully performed, even though the E-vita open stent graft coarctation necessitated another endovascular procedure.
This latter procedure was easily performed because of the presence of the previous open stent. Experience with the frozen elephant trunk technique is limited, but early results ${ }^{1,4,5}$ seem encouraging. Our experience confirms this trend; complex thoracic aortic pathologies such as chronic dissection and chronic degenerative aneurysm involving a large part of the thoracic aorta can be successfully treated with this technique. These are complex and time-consuming operations and good results can be obtained only if good strategies of myocardial, cerebral, and visceral protection are adopted.

In conclusion, the single-stage approach represents an effective surgical option for the treatment of complex aortic disease of the thoracic aorta. Strict monitoring of the patient must be carried out to detect possible complications that may require prompt treatment.

\section{References}

1. Baraki H, Hagl C, Khaladj N, Kallenbach K, Weidemann J, Haverich A, et al. The frozen elephant trunk technique for treatment of thoracic aortic aneurysms. Ann Thorac Surg. 2007;83:S819-31.

2. Pacini D, Leone A, Di Marco L, Marsilli D, Sobaih F, Turci S, et al. Antegrade selective cerebral perfusion in thoracic aorta surgery: safety of moderate hypothermia. Eur J Cardiothorac Surg. 2007;31:618-22.

3. Estrera AL, Miller 3rd CC, Porat EF, Huynh TT, Winnerkvist A, Safi HJ. Staged repair of extensive aortic aneurysm. Ann Thorac Surg. 2002;74:S1803-5; discussion $\mathrm{S} 18$

4. Herold U, Tsagakis K, Kamler M, Massoudy P, Assenmacher E, Eggebrecht H, et al. Change of paradigms in the surgical treatment of complex thoracic aortic disease. Herz. 2006;3:434-42

5. Schoenhoff FS, Schmidli J, Eckstein FS, Berdat PA, Immer FF, Carrel TP. The frozen elephant trunk: an interesting hybrid endovascular-surgical technique to treat complex pathologies of the thoracic aorta. J Vasc Surg. 2007;45: $597-9$. 

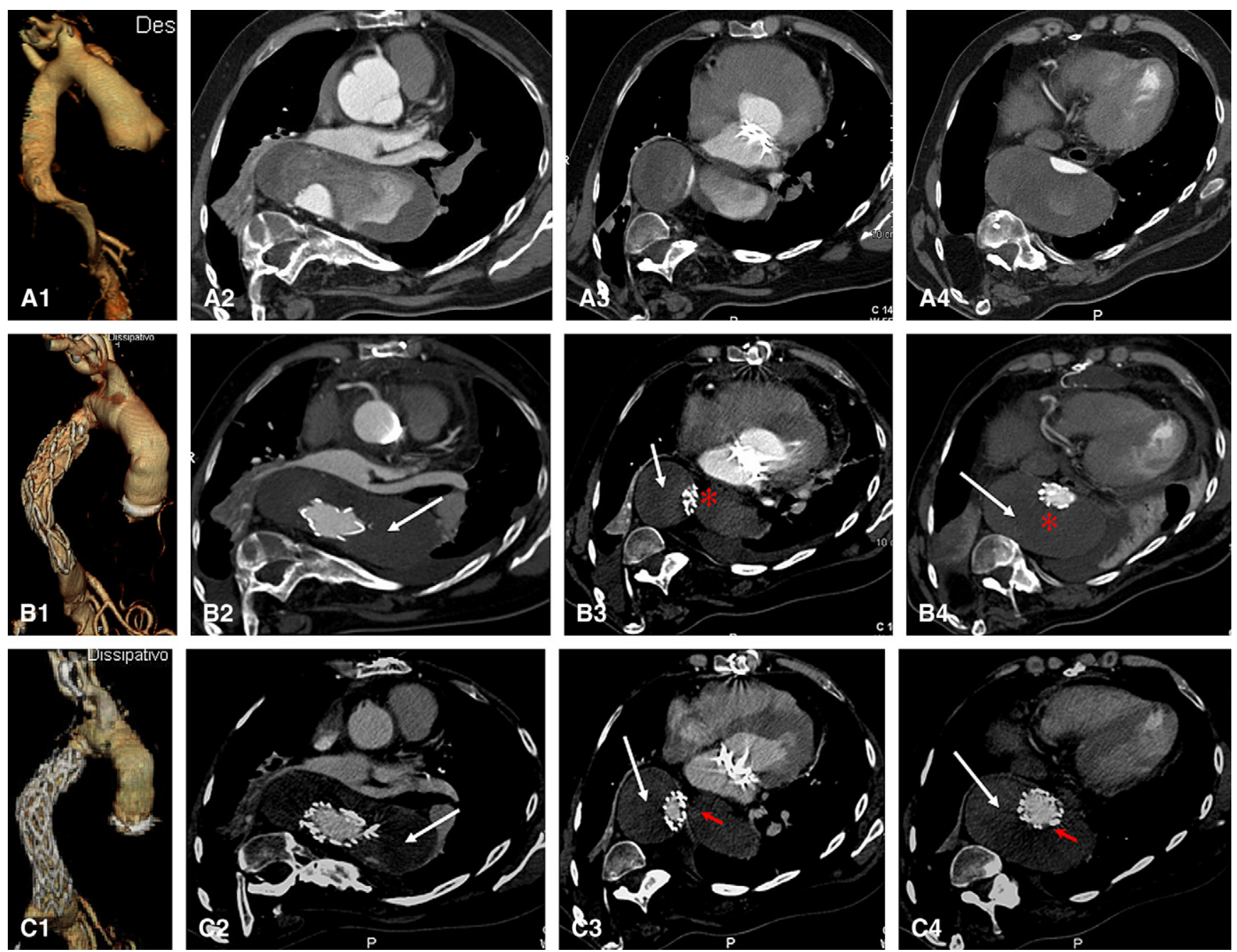

FIGURE E1. Multidetector computed tomographic volume rendering $(A 1, B 1$, and $C 1)$ and axial $(A 2-A 4, B 2-B 4$, and $C 2-C 4)$ images showing dissected thoracic aorta before surgery (A1-A4), after surgical correction with E-vita open prosthesis $(B 1-B 4)$, and after endovascular stent graft extension (C1-C4). Complete thrombosis of false lumen (arrow), partial expansion of E-vita device (asterisk), and stent graft expansion after endovascular extension (arrowhead) are also shown. 